\title{
The analysis of myotonia congenita mutations discloses functional clusters of amino acids within the CBS2 domain and the $\mathrm{C}$-terminal peptide of the $\mathrm{CIC}$ - 1 channel
}

\author{
Concetta Altamura $^{1}$ | Sabrina Lucchiari ${ }^{2,3}$ | Dalila Sahbani ${ }^{1}$ | Gianna Ulzi ${ }^{2,3}$ \\ Giacomo P.Comi ${ }^{2,3}$ | Paola D'Ambrosio ${ }^{4}$ | Roberta Petillo ${ }^{4}$ Luisa Politano ${ }^{4}$
}

Liliana Vercelli $^{5}$ | Tiziana Mongini ${ }^{5}$ | Maria Teresa Dotti ${ }^{6}$ | Rosanna Cardani ${ }^{7}$ |

Giovanni Meola $^{8}$ | Mauro Lo Monaco ${ }^{9,10}$ | Emma Matthews ${ }^{11}$ | Michael G. Hanna ${ }^{11}$ |

Maria Rosaria Carratù $^{12}$ | Diana Conte ${ }^{1}$ | Paola Imbrici ${ }^{1}$ | Jean-François Desaphy ${ }^{12}$

\footnotetext{
${ }^{1}$ Department of Pharmacy-Drug Sciences, University of Bari Aldo Moro, Bari, Italy

${ }^{2}$ Dino Ferrari Centre, Neuroscience Section, Department of Pathophysiology and Transplantation (DEPT), University of Milan, Milan, Italy

${ }^{3}$ Neurology Unit, IRCCS Fondazione Ca' Grande Ospedale Maggiore Policlinico, Milan, Italy

${ }^{4}$ Cardiomyology and Medical Genetics, Department of Experimental Medicine, University of Campania, Naples, Italy

${ }^{5}$ Neuromuscular Unit, Department of Neurosciences, Hospital Città della Salute e della Scienza of Torino, University of Torino, Turin, Italy

${ }^{6}$ Unit of Neurology and Neurometabolic Disorders, Department of Medicine, Surgery and Neurosciences, University of Siena, Siena, Italy

${ }^{7}$ Laboratory of Muscle Histopathology and Molecular Biology, IRCCS Policlinico San Donato, Milan, Italy

${ }^{8}$ Department of Biomedical Sciences for Health, University of Milan, IRCCS Policlinico San Donato, Milan, Italy

${ }^{9}$ Institute of Neurology, Catholic University of Sacred Heart, Polyclinic Gemelli, Rome, Italy

${ }^{10}$ MiA Onlus ("Miotonici in Associazione”), Portici, Italy

${ }^{11}$ MRC Centre for Neuromuscular Diseases, UCL Institute of Neurology and National Hospital for Neurology and Neurosurgery, London, UK

${ }^{12}$ Department of Biomedical Sciences and Human Oncology, University of Bari Aldo Moro, Polyclinic, Bari, Italy
}

Correspondence

Jean-François Desaphy, Department of Biomedical Sciences and Human Oncology, University of Bari Aldo Moro, Polyclinic, Bari, Italy.

Email: Jeanfrancois.desaphy@uniba.it

Funding information

Fondazione Telethon, Grant/Award Number: GGP14096; Association Française contre les Myopathies, Grant/Award Number: 19027; Università degli Studi di Bari Aldo Moro, Grant/Award Number: Fondi Ateneo 2014

Communicated by Garry R. Cutting

This work was supported by Telethon-Italy (grant number GGP14096 to DC), Association Française contre les Myopathies (grant number 19027 to J-FD), and Fondi di Ricerca di Ateneo 2014 of University of Bari (to PI and J-FD).

\begin{abstract}
Myotonia congenita (MC) is a skeletal-muscle hyperexcitability disorder caused by loss-offunction mutations in the $\mathrm{ClC}-1$ chloride channel. Mutations are scattered over the entire sequence of the channel protein, with more than 30 mutations located in the poorly characterized cytosolic C-terminal domain. In this study, we characterized, through patch clamp, seven $\mathrm{CIC}-1$ mutations identified in patients affected by $\mathrm{MC}$ of various severities and located in the $\mathrm{C}$-terminal region. The p.Val829Met, p.Thr832lle, p.Val851Met, p.Gly859Val, and p.Leu861Pro mutations reside in the CBS2 domain, while p.Pro883Thr and p.Val947Glu are in the C-terminal peptide. We showed that the functional properties of mutant channels correlated with the clinical phenotypes of affected individuals. In addition, we defined clusters of CIC-1 mutations within CBS2 and C-terminal peptide subdomains that share the same functional defect: mutations between 829 and 835 residues and in residue 883 induced an alteration of voltage dependence, mutations between 851 and 859 residues, and in residue 947 induced a reduction of chloride currents, whereas mutations on 861 residue showed no obvious change in $\mathrm{CIC}-1$ function. This study improves our understanding of the mechanisms underlying $\mathrm{MC}$, sheds light on the role of the $\mathrm{C}$-terminal region in $\mathrm{ClC}-1$ function, and provides information to develop new antimyotonic drugs.

KEYWORDS

C-terminal, ClC-1, myotonia congenita, patch clamp
\end{abstract}




\section{1 | INTRODUCTION}

Myotonia congenita (MC) (MIM\# 160800) belongs to the group of nondystrophic myotonias and can be inherited either in an autosomal dominant (Thomsen disease) or a recessive manner (Becker disease). Clinically, this disorder is characterized by myotonic discharges at electromyography and delayed muscle relaxation (stiffness), sometimes accompanied by muscle hypertrophy, transient weakness, cramping, and/or pain (Imbrici et al., 2015a; Lehmann-Horn, Jurkat-Rott, \& Rüdel, 2008; Tang \& Chen, 2011). The symptoms are mitigated by mexiletine, a sodium channel blocker that represents the first-choice therapeutic option for MC (Imbrici et al., 2016a; Lo Monaco, D'Amico, Luigetti, Desaphy, \& Modoni, 2015; Statland et al., 2012; Suetterlin et al., 2015). MC is caused by loss-of-function mutations in the CLCN1 gene (MIM\# 118425), encoding the major skeletal-muscle chloride channel $\mathrm{CIC}-1$. The majority of $\mathrm{MC}$ mutations reduce membrane surface channel expression due to defective trafficking or cause changes of channel function including shifts of voltage dependence of protopore and common gating, reduced single channel conductance, or altered ion selectivity (Desaphy et al., 2013; Fahlke, Beck, \& George, 1997; Imbrici et al., 2015a; Pusch, Steinmeyer, Koch, \& Jentsch, 1995; Ronstedt et al., 2015; Ulzi et al., 2012; Vindas-Smith et al., 2016; Warnstedt et al., 2002; Weinberger et al., 2012; Wu et al., 2002). This channel is a homodimer, composed of two identical subunits, each forming a gated pore. Each subunit consists of $18 \alpha$-helices (helices A to R), 17 of which are embedded in the plasma membrane and 2 tandem cystathionine- $\beta$-synthase (CBS) domains are located in the intracellular C-terminal region (Markovic \& Dutzler, 2007; Meyer \& Dutzler, 2006; Park \& MacKinnon, 2018). The CIC-1 channels are the major contributors to the resting membrane conductance, which is crucial for the correct membrane repolarization and propagation of action potential (Imbrici et al., 2015a; Nielsen, De Paoli, Riisager, \& Pedersen, 2017). The myotonic mutations identified up to date are widely distributed across the entire sequence of the channel protein, both in the transmembrane region and in the cytosolic $\mathrm{N}$-terminal and $\mathrm{C}$-terminal parts. The $\mathrm{C}$-terminal cytoplasmic region, including the two CBS domains, is notably large. It contains about 400 of the 991 amino acid residues forming the CIC-1 monomer (Estévez, Pusch, Ferrer-Costa, Orozzo, \& Jentsch, 2004; Hebeisen et al., 2004). Various functional roles have been proposed for this region, such as regulation of $\mathrm{CIC}-1$ trafficking and gating, modulation by intracellular ATP, $\mathrm{NAD}^{+}$, and PKC and sensitivity to external chloride (Bennetts et al., 2005; Bennetts, Yu, Chen, \& Parker, 2012; Hebeisen \& Fahlke, 2005; Hsiao, Huang, Tang, \& Lin, 2010). However, the precise physiological significance remains to be elucidated. Experiments performed in the myotonic goat identified a single-nucleotide mutation in CIC-1 CBS2 (corresponding to $\mathrm{A} 885 \mathrm{P}$ in human $\mathrm{ClC}-1$ ), which causes the symptoms of myotonia and reduces chloride conductance due to the shift of the channel open probability to more positive potentials (Beck, Fahlke, \& George, 1996). Since then, at least 14 missense and 20 nonsense mutations have been found in the C-tail of CIC-1 (Leiden Database): some of these variants have been identified as polymorphisms (e.g., p.Pro727Leu), whereas others were functionally characterized and associated with dominant or recessive myotonic phenotypes
(Brugnoni et al., 2013; Fialho et al., 2007; Liu et al., 2015; Macias et al., 2007).

Here, we provide the functional characterization of seven missense mutations identified in myotonic patients and located in the CBS2 domain (p.Val829Met, p.Thr832Ile, p.Val851Met, p.Gly859Val, and p.Leu861Pro) and C-peptide (p.Pro883Thr and p.Val947Glu) of $\mathrm{hClC}-1$, with the aim of verifying mutation pathogenicity and to gain insight into the role of the C-terminal in $\mathrm{ClC}-1$ function. We found that $\mathrm{CIC}-1$ mutant biophysical defects largely account for the clinical phenotype and identified structural subdomains within the C-terminal region that regulate $\mathrm{ClC}-1$ channel gating and expression.

\section{2 | MATERIALS AND METHODS}

\section{1 | Genetic diagnosis}

Written informed consent for DNA storage and use for genetic analysis and research purposes was obtained from all the patients and relatives, as required by the Ethical Committee of the University of Milan and in accordance with the Declaration of Helsinki. Genomic DNA was extracted from peripheral blood using standard procedures, as previously described (Ulzi et al., 2012). The 23 coding exons and adjacent intronic sequences were amplified by PCR (primers are available upon request), sequenced in an automated sequencer (Applied Biosystem, USA), and compared with Genbank sequence NM_000083. The missense mutations identified in patients were not found in 200 Italian control chromosomes. The new CLCN1 variants were included to the Leiden Open Variation Database (http://www.lovd.nl/3.0/home).

\section{2 | Clinical diagnosis}

We investigated seven Italian patients with clinically and genetically defined MC. All patients were referred to our clinics due to variable grades of muscle stiffness. Neurological examination was specifically conducted to search for myotonic signs, such as muscle weakness, stiffness, and painful myotonia.

\subsection{Site-directed mutagenesis and expression of WT and mutant hClC-1 channels}

Mutations were introduced into the plasmid pcDNA3.1/V5-His TOPO TA Expression Kit (Invitrogen) containing the full-length wild-type (WT) hClC-1 cDNA using the Quickchange TM Site-Directed Mutagenesis Kit (Stratagene, La Jolla, CA, USA). The complete coding region of the cDNA was sequenced to exclude polymerase errors. Human embryonic kidney 293T (HEK293T) cells were transiently transfected with a mixture of the hClC $-1(5 \mu \mathrm{g})$ and CD8 reporter plasmids ( $1 \mu \mathrm{g})$ using the calcium-phosphate precipitation method (Desaphy et al., 2013). Cells were examined between 36 and 80 hours after transfection. Only cells decorated with anti-CD8 antibody-coated microbeads (Dynabeads M450, Invitrogen) were used for patch-clamp recordings.

\subsection{Electrophysiology and data analysis}

Standard whole-cell patch-clamp recordings were performed at room temperature $\left(20^{\circ} \mathrm{C}\right)$ using an Axopatch 200B amplifier (Axon 
Instruments), as previously described (Desaphy et al., 2013; Imbrici et al., 2016b; Tricarico et al., 2013). The composition of the extracellular solution was (in $\mathrm{mM}$ ): $140 \mathrm{NaCl}, 4 \mathrm{KCl}, 2 \mathrm{CaCl}_{2}, 1 \mathrm{MgCl}_{2}$, and 5 Hepes, and the $\mathrm{pH}$ was adjusted to 7.4 with $\mathrm{NaOH}$. The pipette solution contained (in $\mathrm{mM}$ ): $130 \mathrm{CsCl}, 2 \mathrm{MgCl}_{2}, 5 \mathrm{EGTA}$, and 10 Hepes, and the $\mathrm{pH}$ was adjusted to 7.4 with $\mathrm{CsOH}$. In this condition, the equilibrium potential for chloride ions was about $-2.8 \mathrm{mV}$ and cells were clamped at the holding potential (HP) of $0 \mathrm{mV}$. Pipettes were pulled from borosilicate glass and had resistance minor than $2 \mathrm{M} \Omega$, when filled with the above pipette solutions. More than $80 \%$ of series resistance was compensated using the dedicated Axopatch 200B circuit to minimize voltage errors, while recordings showing nonnegligible leak current were discarded. Currents were low-pass filtered at $2 \mathrm{kHz}$ and digitized with sampling rates of $50 \mathrm{kHz}$ using the Digidata 1440A AD/DA converter (Axon Instruments). Chloride currents were recorded $\sim 5$ minutes after achieving the whole-cell configuration, to allow the pipette solution to diffuse into the cell.

Voltage-dependent channel activity was measured by applying specific voltage steps from the HP, as described in the Results section and shown in the figures. We measured the I-V relationship and the overall apparent open probability in high-chloride (134 mM) intracellular solutions to enhance current amplitude. Voltage steps of 400 ms were applied from -150 to $+120 \mathrm{mV}$ in $10 \mathrm{mV}$ intervals, each followed by a voltage step at $-105 \mathrm{mV}$ during which tail currents were recorded. Voltage steps were applied every 3 seconds to allow complete recovery of current amplitude at the HP between two pulses.

The instantaneous and steady-state current-voltage relationships were drawn by measuring instantaneous and steady-state current densities $\left(\mathrm{pA} \mathrm{pF}^{-1}\right)$ at the beginning ( 1 ms) and end ( 390 ms) of each voltage step. Overall apparent open probability (Po) for WT and mutant channels was obtained from normalized peak tail currents measured during the voltage step at $-105 \mathrm{mV}$ reported as a function of the voltage of the preceding pulses (Desaphy et al., 2013). The points were fitted with a Boltzmann function:

$$
P_{\mathrm{o}}(V)=P_{\min }+\left(1-P_{\min }\right) /\left\{1+\exp \left[\left(V-V_{0.5}\right) / k\right]\right\}
$$

where $P_{\min }$ is the minimal value of $P_{0}, V_{0.5}$ is the half-maximal activation potential, and $k$ is the slope factor.

Open probability for common gating ( $P_{\mathrm{o}}$ common) was obtained by using a similar protocol to that for the overall $P_{\mathrm{o}}$ (200 ms conditioning pulse), except that an extra $400 \mu \mathrm{s}$ activation pulse to $+180 \mathrm{mV}$ was added before stepping to $-105 \mathrm{mV}$ (Imbrici et al., 2015b, 2016b). This very positive step fully activates the fast gates of the channel and the tail currents at $-105 \mathrm{mV}$ then reflect only slow gating. Apparent $P_{o}$ total and $P_{o}$ common were calculated from normalized instantaneous current amplitude measured at the beginning of the tail pulse at $-105 \mathrm{mV}$. Because overall apparent open probability equals the product of its protopore and common components, open probability of the protopore gates $\left(P_{o}\right.$ protopore) was then calculated for a given test voltage by dividing the relevant $P_{o}$ by its corresponding $P_{o}$ common. The voltage dependence of channel activation was examined by plotting the apparent open probability as a function of membrane potential and fitting data points with a Boltzmann function.
Data were analyzed off-line by using pClamp 10.3 (Axon Instruments) and SigmaPlot 8.02 (Systat Software $\mathrm{GmbH}$ ) software.

Results are reported as means \pm SEM from $n$ cells, and statistical analysis was performed using Student's test, with $P<0.05$ considered as significant.

\section{5 | Homology model}

To describe the position of the MC CIC-1 mutations in Figure 1, we used the homology model of the dimeric $\mathrm{ClC}-1$ channel based on the crystallographic coordinates of the eukaryotic $\mathrm{Cl}-/ \mathrm{H}+$ exchanger CmClC (PDB id: 3ORG; Bennetts \& Parker, 2013; Feng, Campbell, Hsiung, \& MacKinnon, 2010).

\section{3 | RESULTS}

\subsection{Clinical phenotype of MC families}

Myotonic patients were examined in various Italian clinical centers, except those carrying the Pro883Thr mutation, who were referred to the National referral center for skeletal-muscle channelopathies in the UK.

Mutation 1: p.Val829Met. A c.2485G >A transition (p.Val829Met) was identified in a male patient, now 52 years old, with late-onset myotonia characterized by lower-limb stiffness and myalgia. The CK levels were normal (300 UI/L). On his last visit, 6 months ago, he showed autonomous walking, climbing, and descending stairs without support, rising from the chair without specific support. No muscle deficit was detected on muscle examination. Modest hands myotonia was evident whereas eyelid myotonia was not detected. The proband also showed mild hypotrophy in the proximal internal area of the thigh bilaterally. Control ECG was normal. Molecular testing resulted negative for myotonic dystrophy type 2 (DM2). The clinical phenotype was compatible with Thomsen disease. He takes mexiletine $400 \mathrm{mg}$ a day with a clear benefit on stiffness and cramps; in the past, he took quinine with partial benefit and clonazepam, gabapentin, or pregabalin that were instead poorly efficacious.

Mutation 2: p.Thr832lle. The c.2495C $>$ T recessive mutation in CLCN1, producing the p.Thr832lle amino acid substitution in CIC-1 protein, was detected in one male patient, in compound heterozygosity with the already known c.180+3A $>$ T mutation. The proband presented at the age of 20 with lower-limb muscle stiffness and muscle hypertrophy. Neurologic examination revealed mild myotonia with warm-up, whereas electromyography showed myotonic discharges in all the investigated muscles. The patient is under mexiletine treatment (600 mg a day) with great reduction of myotonic episodes. The parents, both asymptomatic, come from neighboring villages in the province of Avellino and carry one of the two mutations identified in the proband, suggesting an autosomal recessive inheritance. The patient is the second of two children; the older brother is healthy.

Mutation 3: p.Val851Met. Two related patients were found to carry the c.2551G $>$ A (p.Val851Met) mutation in combination with c.86A>C (p.His29Pro) on the same chromosome. This latter mutation was 


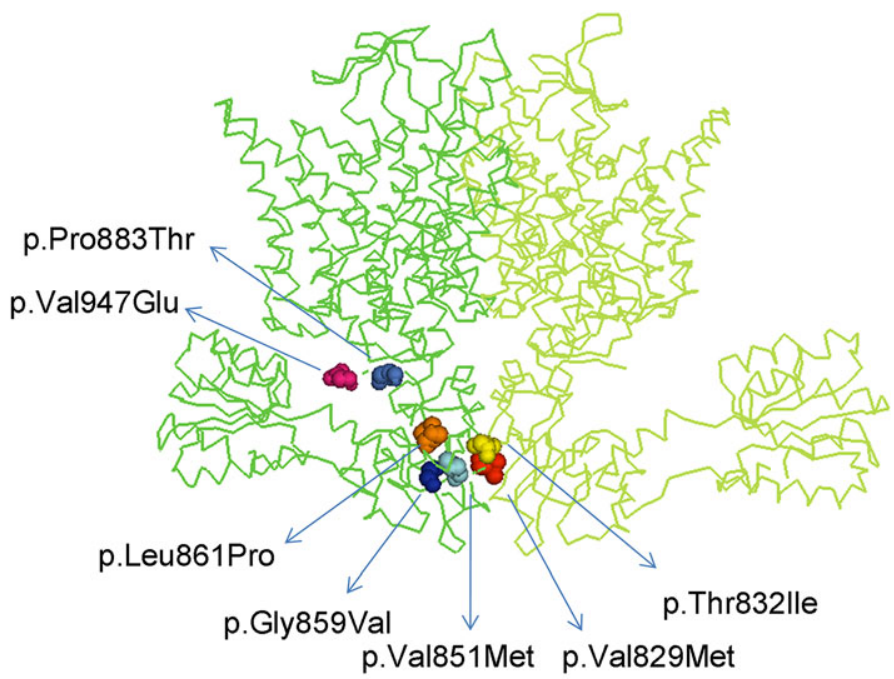

\begin{tabular}{|c|c|c|c|c|c|}
\hline \multicolumn{6}{|c|}{ pecies-specific ClC-1: } \\
\hline Rat & QLVEQTTLHKTHTLFS LLGLHLAYVTSM-GKLRGVLALE & 870 & RPPLAS & 892 & PARVEGE \\
\hline louse & QLVEQTTLHKTHTLFSLLGLHLAYVTSM-GKLRGVLALE & 870 & RPPLAS & 892 & PARAEGE \\
\hline Dog & QLVEQTSLHKTHTLFSLLGLHLAYVTSM-GKLRGVLALE & 789 & RPPLAS & 811 & PAKVEGE \\
\hline \multicolumn{6}{|c|}{ Human CLC members: } \\
\hline $\mathrm{ClC}-2$ & QLVERTSLHKTHTIFSLLGVDHAYVTS & 833 & RPPLAS & 856 & \\
\hline $\mathrm{ClC}-\mathrm{Ka}$ & TLFSETTLHQAQNLFKLLNLQSLFVTSR-GRAVGCVSWV & 671 & NPPAPK & 687 & -- \\
\hline $\mathrm{ClC}-\mathrm{Kb}$ & KLSPETSLHEAHNLFELLNLHSLFVTSR-GRAVGCVSWV & 671 & NPPAPK & 687 & --- \\
\hline $\mathrm{ClC}-3$ & TVTDHT PMEIVVDI FRKLGLRQCLVTHN-GRLLGI ITKK & 798 & QDPASI & 815 & -- \\
\hline $\mathrm{IC}-4$ & TVTDHTPMETVVDI FRKLGLRQCLVTRS-GRLLGI ITKK & 740 & QDPESI & 757 & ------- \\
\hline $1 C-5$ & TVTDLT PMEIVVDI FRKLGLRQCLVTHN-GRLLGI ITKK & 726 & QDPDSI & 743 & ------- \\
\hline $\mathrm{ClC}-6$ & TVSPNTHVSQVFNLFRTMGLRHLPVVNAVGEIVGI ITRH & 850 & RQHYQT & 868 & ------ \\
\hline $\mathrm{ClC}-7$ & TVPQEASLPRVFKLFRALGLRHLVVVDNRNQVVGLVTRK & 784 & LEELSL & 802 & ---- \\
\hline
\end{tabular}

FIGURE 1 Structure and alignment of CIC channels. (A) Three-dimensional representation of hCIC-1 channel modeled upon the X-ray structure of an eukaryotic $\mathrm{Cl}^{-} / \mathrm{H}^{+}$exchanger $\mathrm{CmClC}$ (Feng et al., 2010) showing the localization of the C-terminal MC mutations. (B) Amino acid alignment of CLC proteins

previously found in association with p.Tyr257X and p.Ala566Val in another patient reporting moderate myotonia, permanent limb-girdle muscle weakness, and scoliosis (Skálová et al., 2013). The proband is a woman, now 50 years old, who presented with lower-limb muscle weakness, stiffness with difficulty to start movements, and painful myotonia and warm-up. Symptoms initiated at 16 years old. CK level was in the standard range. Neurological examination showed muscular hypertrophy with particular involvement of femoral quadriceps and gastrocnemius muscles. Myotonia was reported for the lower limbs and for the hands and face muscles, and worsened with cold. Myotonic myopathy was also evident on EMG. The clinical phenotype was suggestive of Thomsen disease. At the time of examination, the patient was taking thiocolchicoside and magnesium pidolate. Concurrent disorders were asthma, hypercholesterolemia, esophagitis, and tachycardia. Therapy includes pantoprazole, diltiazem, olmesartan medoxomil, flutiform (a combination of fluticasone and formoterol), ebastina, montelukast, and cholecalciferol.

Her father, now 77 years old, noticed weakness, stiffness, myopathy, and painful myotonia at the age of six. In addition to p.Val851Met and p.His29Pro in cis, he carries the known intronic mutation c.116710T>C (Trip et al., 2008). Myotonic myopathy was also observed in proband's sister and first-born son of the latter.

Mutation 4: p.Gly859Val. The c.2576G $>T$ substitution (p.Gly859Val) was identified in a male patient, now 42 years old, with onset during early infancy, characterized by generalized muscle stiffness and hypertrophy. Myotonia develops mainly at the beginning of movement after prolonged rest and improves with exercise. The EMG studies disclosed myotonic discharges in all the examined muscles, while neurophysiologic test showed a transitory depression of CMAP. Mexiletine was started at $400 \mathrm{mg} /$ day and was then incremented to $600 \mathrm{mg} /$ day with a dramatic reduction of myotonia (Ginanneschi et al., 2017).

Mutation 5: p.Leu861Pro. A c.2582T>C transition (p.Leu861Pro) was detected in a male patient who showed a very severe form of myotonia, especially characterized by transitory weakness, suggestive of Becker myotonia. Indeed, the mutation was found in compound heterozygosity with the well-known c.568GG > TC transition (p.Gly190Ser) (Brugnoni et al., 2013; Desaphy et al., 2013; Shalata et al., 2010). Proband's father also carries p.Leu861Pro mutation, but his clinical and neurophysiological examinations resulted negative for myotonia. The mother, carrying p.Gly190Ser mutation, showed mild myotonic symptoms nearly limited to lower limbs. She was considered as affected by Thomsen's myotonia. She always refused any antimyotonic treatment. The proband is treated with mexiletine $600 \mathrm{mg} / \mathrm{day}$ since 2005 with good but not dramatic improvement. The $3-\mathrm{Hz}$ repetitive nerve stimulation (Lo Monaco et al., 2015) induced a 93\% compound muscle action depression before treatment that remained always above $40 \%$ during treatment.

Mutation 6: p.Pro883Thr. The c.2647C >A (p.Pro883Thr) was found in homozygosity in three unrelated individuals, two of them 
were previously reported (Fialho et al., 2007). They were all referred for genetic testing with a clinical query of $M C$ without any further details. In one case, the father, who was also reported as symptomatic, was homozygous for the same mutation. The mother was heterozygous, although she also reported some myotonic symptoms. Unfortunately, no further clinical details are available.

Mutation 7: p.Val947Glu. The c.2840T>A substitution (p.Val947Glu) was identified in a male patient, now 49 years old, who showed his first myotonic symptoms at the age of 18 . This mutation was found in compound heterozygosity with a novel mutation, p.Thr533lle. Neurological examination revealed cold-aggravated myotonia, mainly in hands, lower-limb muscle stiffness, and generalized muscle hypertrophy.

Figure 1 shows the position of the MC mutations in the human CIC-1 protein model based upon the X-ray crystal structure of a eukaryotic CLC transporter from the thermophilic red alga Cyanidioschyzon merolae (CmCLC) (Feng et al., 2010), and the C-terminal amino acid alignments of CIC-1 from various species and human CLC proteins.

\subsection{Electrophysiological studies of myotonic mutations in $\mathrm{C}$-terminal region}

Chloride currents were recorded in HEK293 cells transfected with the same amount of either WT or mutant hClC-1 chloride channels, using a voltage protocol that allows the determination of $\mathrm{I}-\mathrm{V}$ relationships as well as the voltage dependence of activation (apparent open probability). Chloride currents were recorded using a high-chloride intracellular solution (134 mM), which allows to clearly distinguish the kinetics of WT and mutant channels (Figure 2A).

In these experimental conditions, the p.Val829Met currents were very similar to WT currents. The I-V relationships for instantaneous current density were merely superimposed for WT and p.Val829Met, except for a $35 \%$ reduction of steady-state chloride current density close to the resting membrane potential of muscle cells $(-90 \mathrm{mV})$ (Figure 2B,C). Conversely, both instantaneous and steady-state current amplitudes were greatly reduced through the entire voltage range for p.Thr832lle and p.Val947Glu channels, compared with WT (Figure 2B,C). Different from WT currents, p.Pro883Thr mutant showed a reduction of the instantaneous current amplitudes and a peculiar strong outward rectification (Figure 2B,C).

The analysis of the apparent open probability revealed no significant difference for $\mathrm{p}$.Val947Glu half-maximum activation voltage with respect to WT. In contrast, the voltage dependence of activation of p.Pro883Thr appeared significantly shifted by about $55 \mathrm{mV}$ toward positive potentials. The voltage dependence of activation of p.Val829Met and p.Thr832lle mutants resulted right shifted by about $20 \mathrm{mV}$ (Figure 2D and Table 1). In order to gain insight into the pathogenic mechanism behind these mutations, we also analyzed the effect of the p.Pro883Thr on the protopore and common gating of the channel. Interestingly, this mutation right shifted the voltage dependence for common gating by $\sim 50 \mathrm{mV}$ compared with WT (Supporting Information Figure S1).
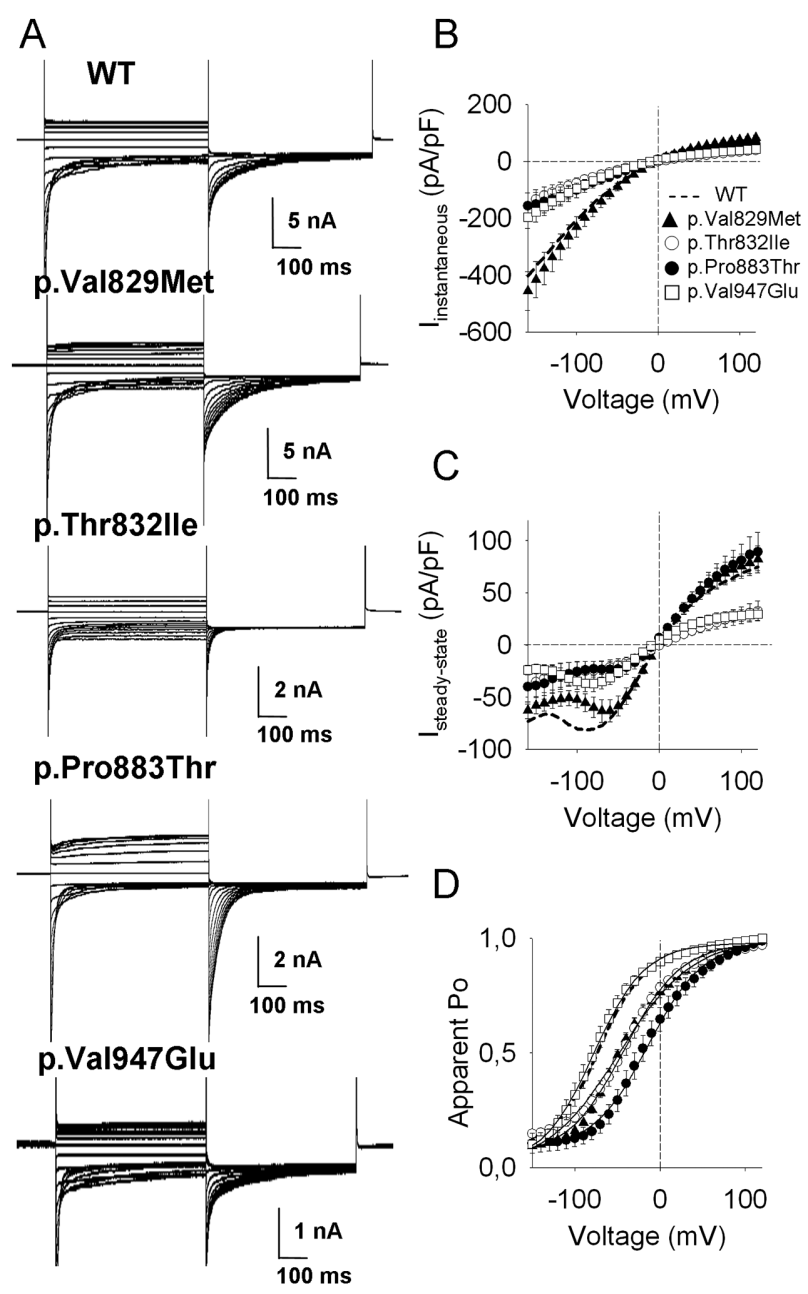

C
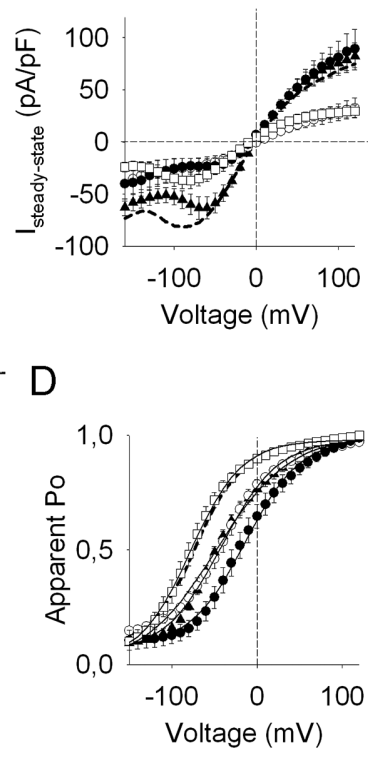

FIGURE 2 Functional characteristics of myotonic hClC-1 channel mutants located in the CBS2 domain and C-terminal peptide. (A) Typical chloride currents recorded in HEK293T cells transfected with WT, p.Val829Met, p.Thr832lle, p.Pro883Thr, or p.Val947Glu hClC-1 variants. Cells were held at $0 \mathrm{mV}$ and $400 \mathrm{~ms}$ voltage pulses were applied from -150 to $+120 \mathrm{mV}$ in $10-\mathrm{mV}$ intervals every 3 seconds. For clarity, only current traces obtained every $20 \mathrm{mV}$ are shown. (B) The instantaneous currents for the indicated channels were measured at the beginning of test voltage pulses, normalized with respect to cell capacitance $(\mathrm{pA} / \mathrm{pF})$, and reported as a function of voltage. (C) Steadystate currents were measured at the end of test voltage pulses and reported as mean current density \pm SEM in function of voltage. (D) The voltage dependence of activation was determined by plotting the apparent open probability (Po), calculated from tail currents measured at $-105 \mathrm{mV}$, as a function of test voltage pulses. The relationships obtained from averaged data were fit with a Boltzmann equation, and fit parameters are reported in Table 1

\subsection{Coexpression experiments of $p$.Leu861Pro and p.Gly190Ser}

The new recessive mutation, p.Leu861Pro, residing in the CBS2 domain, was associated in compound heterozygosity with the previously characterized p.Gly190Ser mutation (Desaphy et al., 2013; Portaro et al., 2015). Chloride currents carried by homomeric p.Leu861Pro channels were similar to WT currents (Figure 3A). The current amplitude of instantaneous and steady-state chloride currents 
TAB LE 1 Biophysical parameters of hCIC-1 WT and MC mutants

\begin{tabular}{|c|c|c|c|c|c|}
\hline hClC-1 & $\begin{array}{l}\mathrm{SSC}(-90 \mathrm{mV}) \\
(\mathrm{pA} / \mathrm{pF})\end{array}$ & $\begin{array}{l}\mathrm{SSC}(+60 \mathrm{mV}) \\
(\mathrm{pA} / \mathrm{pF})\end{array}$ & $V_{0.5}(m V)$ & $K(\mathrm{mV})$ & $n$ cell \\
\hline WT & $-81.6 \pm 17$ & $52.1 \pm 10$ & $-68 \pm 0.5$ & $26.8 \pm 0.4$ & 18 \\
\hline p.Val829Met & $-55.2 \pm 8.6$ & $58.1 \pm 9$ & $-42 \pm 1.5$ & $33.3 \pm 1.4$ & 18 \\
\hline p.Thr832lle & $-25.1 \pm 9.6$ & $19.2 \pm 5.6$ & $-48.3 \pm 1.9$ & $42.9 \pm 1.5$ & 5 \\
\hline p.Leu861Pro & $-68.4 \pm 14.8$ & $54 \pm 14$ & $-69.3 \pm 1$ & $36.4 \pm 0.8$ & 10 \\
\hline p.Gly190Ser & $-15.7 \pm 3 *$ & $19.2 \pm 5.3 *$ & $160 \pm 5.5 *$ & $43.7 \pm 1.7$ & 5 \\
\hline p.Leu861Pro+p.Gly190Ser & $-39.6 \pm 15$ & $60.7 \pm 22$ & $113.8 \pm 3.9 *$ & $68.2 \pm 2.1$ & 5 \\
\hline WT+p.Gly859Val & $-60.2 \pm 13$ & $29.8 \pm 6$ & $-82 \pm 2.1$ & $29.8 \pm 1.4$ & 12 \\
\hline WT+ p.Val851Met & $-78.4 \pm 30$ & $34 \pm 14$ & $-80.6 \pm 2.2$ & $28.5 \pm 1.6$ & 5 \\
\hline
\end{tabular}

Data are means \pm SEM of the indicated number of cells. SSC, steady-state current measured at -90 and $+60 \mathrm{mV}$. $\mathrm{V}_{0.5}$, half-maximal activation potential; $k$, slope factor; ${ }^{*} \mathrm{P}<0.05$ or less compared with WT.
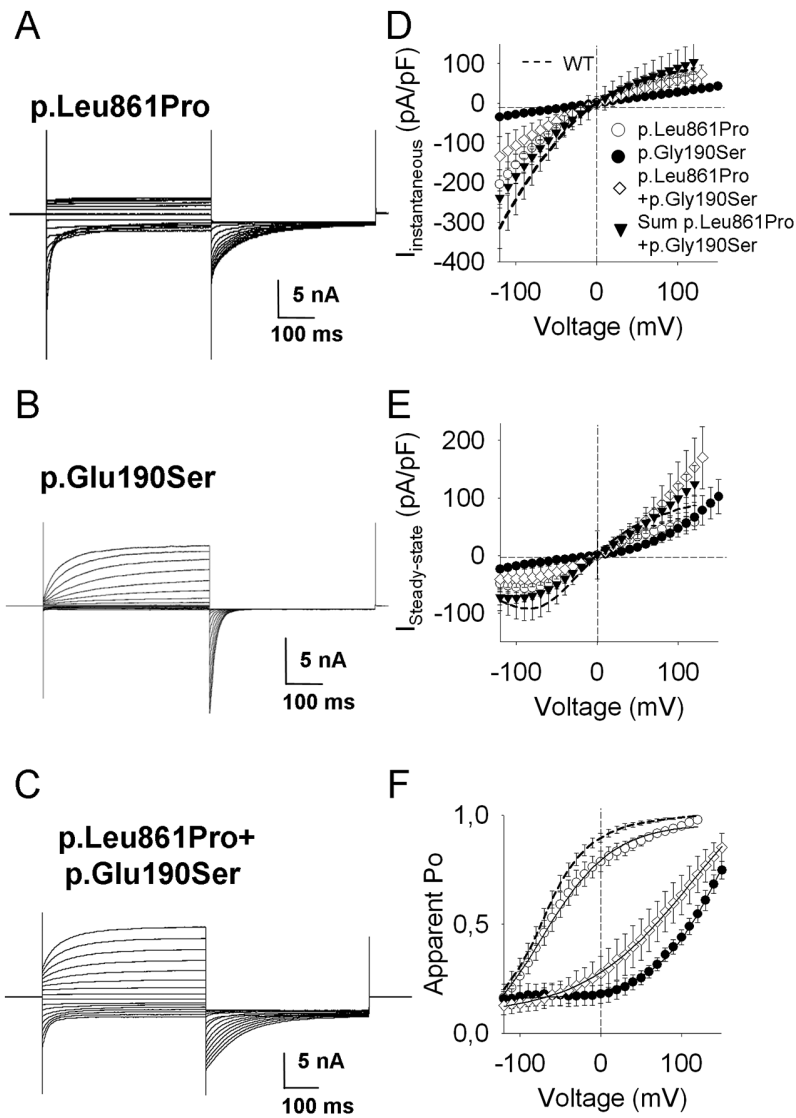

FIGURE 3 Functional characteristics of p.Leu861Pro, p.Gly190Ser and coexpressed p.Leu861Pro+ p.Gly190Ser hCIC-1 channels. (A-C) Typical chloride currents recorded in HEK293T cells transfected with $5 \mu \mathrm{g}$ p.Leu861Pro or p.Gly190Ser mutant alone, or cotransfected with $5 \mu \mathrm{g}$ p.Leu861Pro and $5 \mu \mathrm{g}$ p.Gly190Ser mutants. (D) The instantaneous currents were measured as in Figure 1B, for p.Leu861Pro, p.Gly190Ser, and p.Leu861Pro+p.Gly190Ser channels. (E) Steady-state currents were measured as described in Figure 2C. (F) The voltage dependence of activations, determined as in Figure 2D, were fitted with a Boltzmann function. Fit parameters are reported in Table 1. Dashed line represents the WT currents for p.Leu861Pro were slightly, albeit not significantly, reduced compared with WT channels, and the voltage dependence of activation of both channels were superimposed (Figure 3A-C and Table 1). As previously shown (Desaphy et al., 2013), p.Gly190Ser induced a dramatic $\sim 260 \mathrm{mV}$ shift of the open probability toward positive voltages, resulting in zero chloride current within the physiological voltage range of sarcolemma.

Cotransfection experiments were performed with the same amount $(5 \mu \mathrm{g})$ of p.Leu861Pro and p.Gly190Ser plasmids. The resulting chloride currents showed properties recapitulating both p.Gly190Ser and p.Leu861Pro chloride currents: deactivating currents at negative voltages and tail currents were similar to p.Leu861Pro, whereas slowly activating outward currents at positive voltages were similar to p.Gly190Ser (Figure 3A-C). Nevertheless, the instantaneous and steady-state current densities of coexpressed channels were, at each voltage, lower than the algebraic sum current densities of $p$.Leu861Pro and p.Gly190Ser expressed alone, suggesting a dominant-negative effect of p.Gly190Ser mutation on p.Leu861Pro. In addition, the $P_{\mathrm{o}}$ was shifted by $\sim 230 \mathrm{mV}$ toward more positive potentials compared with that of p.Leu861Pro homomeric channels (Figure 3C and Table 1). Thus, heteromeric p.Leu861Pro+p.Gly190Ser channels were less likely to be open within the physiological voltage range.

\subsection{Coexpression experiments of WT and p.Gly859Val or p.Val851Met}

Two mutations sited in CBS2 domain, p.Gly859Val and p.Val851Met, were inherited in a dominant manner. These residues are well conserved among species and among human CLC protein isoforms (Figure 1). Using MutPred software, p.Val851Met and p.Gly859Val were, respectively, scored with 0.7 and 0.9 probability to be deleterious. According to these results, p.Val851Met and p.Gly859Val mutants did not generate any chloride currents in transfected HEK cells within the voltage range of -150 to $+150 \mathrm{mV}$ (Figures $4 \mathrm{~A}$ and $5 \mathrm{~A}$ ).

In order to test whether these mutations exerted a dominantnegative effect on wild-type ClC-1 subunits, we coexpressed p.Val851Met and p.Gly859Val mutants $(5 \mu \mathrm{g})$ with equal amounts 


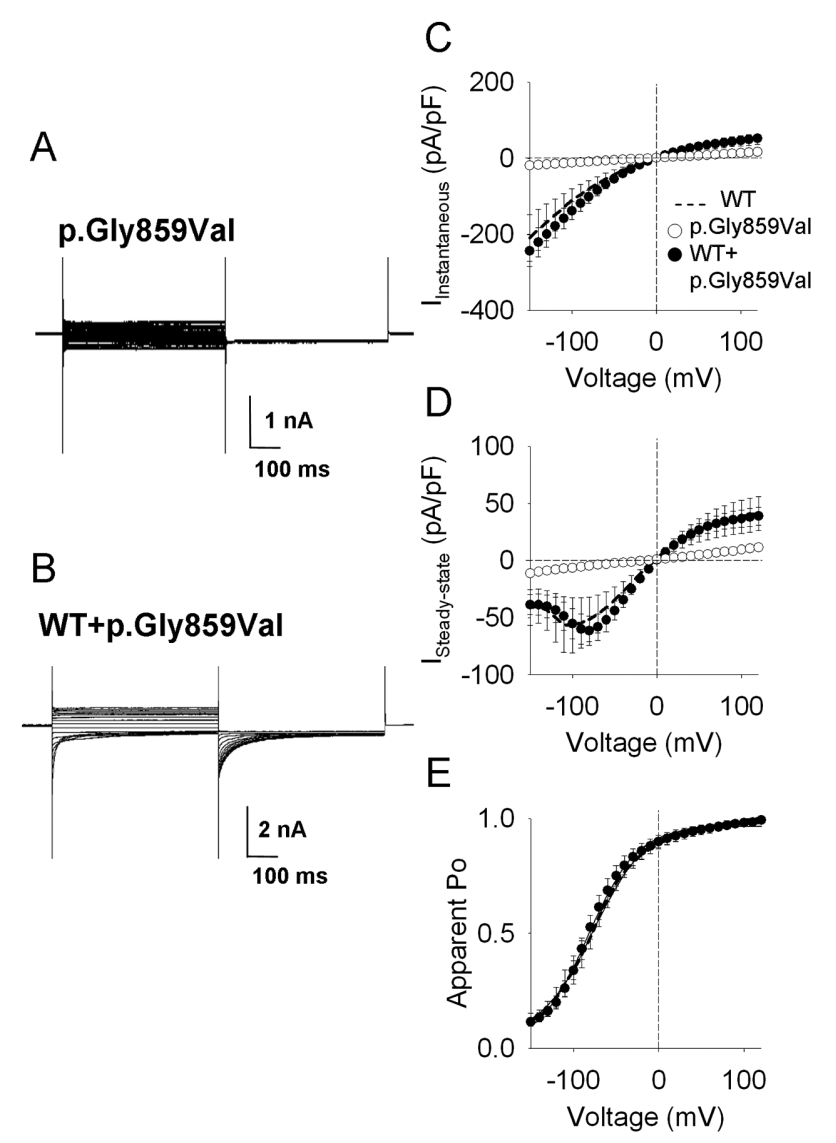

FIGURE 4 Functional characteristics of p.Gly859Val and coexpressed WT+p.Gly859Val hClC-1 channels. (A-B) Typical chloride currents recorded in HEK293T cells transfected with $5 \mu \mathrm{g}$ of p.Gly859Val mutant alone or cotransfected with $5 \mu \mathrm{g} \mathrm{WT}$ and $5 \mu \mathrm{g}$ p.Gly859Val. (C) The instantaneous currents were measured as in Figure 1B, for WT, p.Gly859Val, and WT+p.Gly859Val channels. (D) Steady-state currents were measured as described in Figure $2 \mathrm{C}$. (F) The voltage dependence of activation, determined as in Figure $2 \mathrm{C}$, was fitted with a Boltzmann function. Fit parameters are reported in Table 1

of WT CIC-1 and examined current density and voltage dependence. The chloride currents generated in cells transfected with $5 \mu \mathrm{g}$ of WT ClC-1 alone were considered for comparison. Both WT+p.Val851Met (Figure 5) and WT+p.Gly859Val (Figures 4B and 5B) channels showed gating properties similar to WT, with both inwardly and outwardly rectifying currents. The instantaneous and steady-state currents generated by either coexpressed channels or homomeric WT were superimposed, suggesting that the p.Val851Met and p.Gly859Val pore mutant subunits did not exert a measureable dominant-negative effect on WT in coexpression experiments (Figures 4C-E and 5C-E).

\section{4 | DISCUSSION}

In this study, we report a thorough characterization of newly identified myotonic mutations located in the $\mathrm{C}$-terminal of the $\mathrm{CIC}-1$ channel and associated with different grades of myotonic symptoms that provide

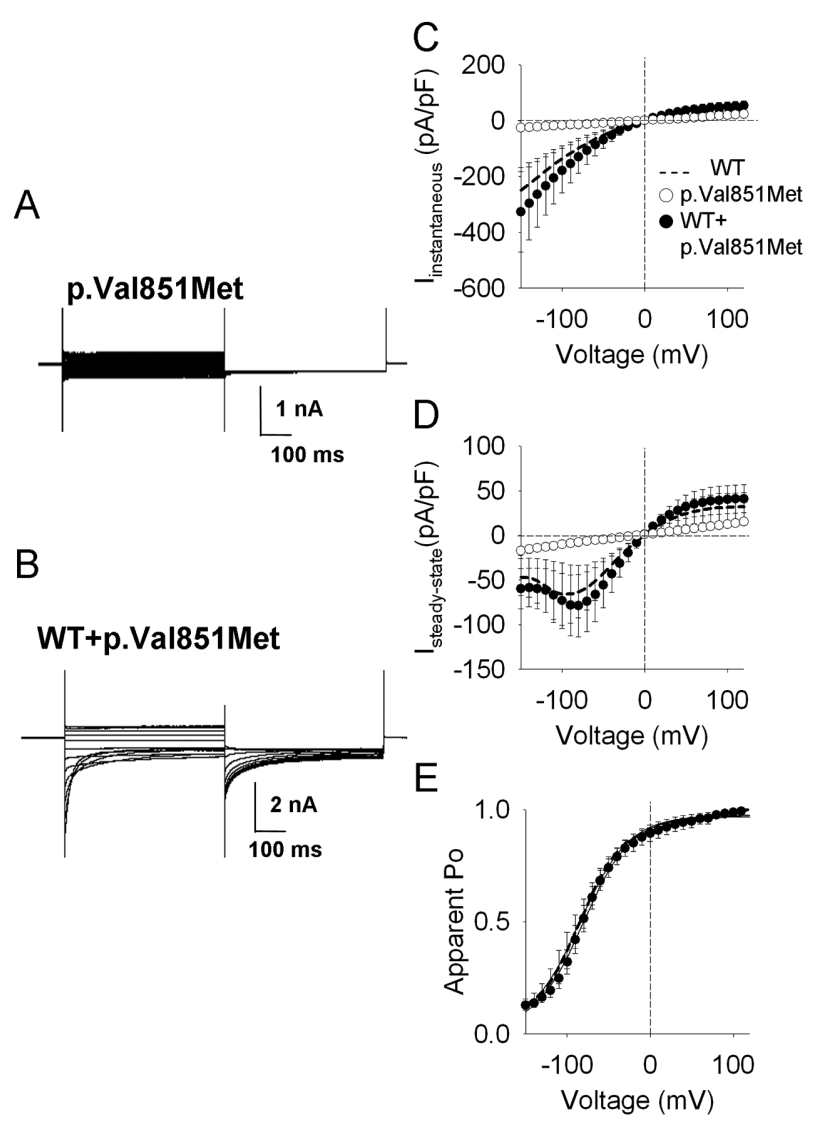

FIGURE 5 Functional characteristics of p.Val851Met and coexpressed WT+p.Val851Met hClC-1 channels. (A-B) Typical chloride currents recorded in HEK293T cells transfected with $5 \mu \mathrm{g}$ p.Val851Met mutant alone or cotransfected with $5 \mu \mathrm{g}$ WT and $5 \mu \mathrm{g}$ p.Val851Met mutant. (C) The instantaneous currents were measured as in Figure 1B, for WT, p.Val851Met, and WT+p.Val851Met channels. (D) Steady-state currents were measured as described in Figure 2C. (F) The voltage dependence of activations, determined as in Figure 2D, were fitted with a Boltzmann function. Fit parameters are reported in Table 1

relevant information about the contribution of the $\mathrm{C}$-terminal domain to $\mathrm{CIC}-1$ activity.

\section{1 | Genotype-phenotype correlation}

The clinical examination of patients carrying p.Val851Met and p.Gly859Val suggested a Thomsen's phenotype associated with moderate myotonia and mild muscle stiffness, which was consistent with the functional analysis of mutant channels. When expressed in HEK cells, these mutants did not produce any chloride currents, confirming their pathogenicity in producing myotonia. Despite such a drastic effect, coexpression experiments performed to reproduce the heterozygous condition of the affected patients demonstrated that p.Val851Met and p.Gly859Val mutations did not exert any dominantnegative effect on WT CIC-1 subunits, thereby ensuring at least half of the sarcolemma chloride conductance. This suggests that WT subunit might temper the severe biophysical defect of individual mutant channels, thus explaining the mild myotonic phenotype of affected carriers (Table 2). 
TABLE 2 Genotype-phenotype correlation

\begin{tabular}{|c|c|c|c|c|}
\hline Genotype & Inheritance & Clinical phenotype & Therapy & Functional defect \\
\hline p.Val829Met & $A D$ & Mild with muscle stiffness and pain & Mexiletine 400 mg/day & $\begin{array}{l}\text { Small voltage-dependent } \\
\text { shift }\end{array}$ \\
\hline p.Thr832lle & $\mathrm{AR}(\mathrm{c} .180+3 \mathrm{~A}>\mathrm{T})$ & $\begin{array}{l}\text { Mild with muscle stiffness, } \\
\text { hypertrophy and warm-up }\end{array}$ & Mexiletine 600 mg/day & $\begin{array}{l}\text { Small voltage-dependent } \\
\text { shift }\end{array}$ \\
\hline p.Val851Met & AD (p.His29Pro in cis) & $\begin{array}{l}\text { Mild with muscle stiffness and pain, } \\
\text { hypertrophy, weakness and } \\
\text { warm-up }\end{array}$ & $\begin{array}{l}\text { Thio-colchicoside, } \\
\text { magnesium pidolate }\end{array}$ & $\begin{array}{r}\text { Nonfunctional channels w/o } \\
\text { dominant-negative effect }\end{array}$ \\
\hline p.Leu861Pro & AR (p.Gly190Ser) & Severe with muscle weakness & Mexiletine $600 \mathrm{mg} / \mathrm{day}$ & $\begin{array}{l}\text { Similar to WT but } \\
\text { dominant-negative effect } \\
\text { with p.Gly190Ser }\end{array}$ \\
\hline p.Pro883Thr & Homozygous & n.a. & n.a. & $\begin{array}{l}\text { Large voltage-dependent } \\
\text { shift }\end{array}$ \\
\hline
\end{tabular}

n.a., information not available.

The p.Val947Glu mutation was identified in a male patient who showed a mild form of MC. Functional characterization revealed that p.Val947Glu mutant channels showed a $60 \%$ reduction of chloride currents, although the voltage dependence resulted similar to WT. The mutation was inherited in compound heterozygosity with p.Thr533lle, a mutation never characterized before. p.Thr533lle is located at the helix $\mathrm{O}$ of the human $\mathrm{ClC}-1$ and has a medium likelihood of being deleterious as predicted by MutPred Software analysis (score 0.54). Other mutations near this residue are associated with a myotonic phenotype, such as p.Ala531Val (Desaphy et al., 2013; Lee et al., 2013), p.Ala535Asp, and p.Val536Leu (Brugnoni et al., 2013), thus suggesting the importance of this region for proper $\mathrm{ClC}$-1 function and supporting the probable pathogenicity of p.Thr533lle. The coexistence of p.Val947Glu and p.Thr533lle in the proband is therefore likely responsible for the mild myotonic phenotype.

Two other mutations in the CBS2 domain were associated with mild myotonic symptoms, p.Val829Met and p.Thr832lle. When expressed in HEK293T cells, p.Val829Met and p.Thr832lle did not change chloride current density but induced a slight shift of voltage dependence toward positive potential. Such an effect may be sufficient to recapitulate the mild clinical phenotype found in the patients.

The p.Leu861Pro variant located in the CBS2 domain was associated with a severe phenotype and was scored with a 0.8 probability to be deleterious (mutPred). The residue is conserved in $\mathrm{ClC}-1$ from different species, though the sequence stream where it is located varies among human CLC protein paralogs. The functional characterization of p.Leu861Pro mutant revealed that current amplitude and voltage dependence were very similar to those of WT protein, thus questioning its pathogenicity. Indeed, the proband's father carrying this mutation in heterozygosity was asymptomatic. Besides p.Leu861Pro, the proband also carries the well-known p.Gly190Ser mutation (Desaphy et al., 2013; Portaro et al., 2015). Coexpression experiments with p.Leu861Pro and p.Gly190Ser produced chloride currents smaller than those of p.Leu861Pro alone, suggesting a dominant-negative effect of p.Gly190Ser on the p.Leu861Pro subunit. It is thus likely that the severity of myotonia in the proband may stem from such a specific dominant-negative effect exerted by the p.Gly190Ser subunit on the p.Leu861Pro subunit. Interestingly, we previously did not observe a dominant-negative effect of p.Gly190Ser on the WT subunit (Portaro et al., 2015), in agreement with the mild myotonic phenotype observed in the proband's mother carrying only p.Gly190Ser. Overall, these results suggest that p.Leu861Pro might act as a disease modifier able to worsen the phenotype caused by the coexisting p.Gly190Ser pore mutation without being pathogenic by itself.

\subsection{ClC-1 structure-function relationship}

The role of the C-terminal domains of CLC channels was investigated through the study of point mutations identified in this region associated with several inheritable diseases (Koch et al., 1992; Kornak et al., 2001; Simon et al., 1997). Deletions, truncations, or missense mutations in the $\mathrm{C}$-tail resulted in poor expression, altered gating, or nonfunctional channels, demonstrating its physiological relevance (Ma, Rychkov, Hughes, \& Bretag, 2009).

In hClC-1, the CBS2 domain encompasses residues 820 to 871 (Estévez et al., 2004; Hebeisen et al., 2004) and has been predicted to play a role in channel gating. Indeed, structural studies on $\mathrm{ClC}$ 1 suggested that the long intracellular C-terminal portion is folded in such a manner that the CBS2 domain may directly interact with the $\alpha$-helix $\mathrm{R}$ containing Tyr578 residue, which participates directly in $\mathrm{Cl}^{-}$coordination during transport (Feng et al., 2010; Imbrici et al., 2015b). Moreover, several mutations occurring in CBS2 have been shown to cause changes in channel voltage dependence, and a mutant with a particularly strong effect, p.His835Arg, displayed a dominantnegative effect on WT in coexpression studies, suggesting an alteration of the common gate (Estévez et al., 2004). Consistent with a role of CBS2 in CIC-1 gating, both p.Val829Met and p.Thr832lle mutations, falling within CBS2, showed a positive shift of channel voltage dependence. Two other neighboring mutations located in CBS2 and well conserved among CLC proteins, p.Val851Met and p.Gly859Val, failed to 


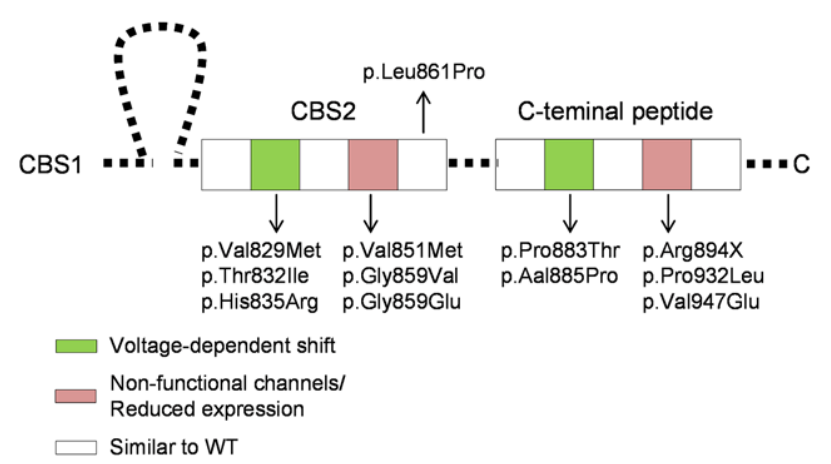

FIGURE 6 Clustering of MC mutations. Schematic drawing showing clusters of $\mathrm{ClC}-1$ mutations within CBS2 and C-terminal peptide subdomains that share the same functional defect

generate chloride current, suggesting either nonfunctional channels or poor expression due to a defect in protein translocation to the membrane. Interestingly, p.Gly859Asp is also associated with a lack of current, suggesting presumably a similar disruption of the CBS domain structure (Bennetts et al., 2005).

In contrast, p.Leu861Pro mutation did not alter CIC-1 function, showing a behavior similar to WT. We cannot exclude that the mutation may affect the channel regulation by intracellular metabolites. Indeed, previous studies have shown that the $\mathrm{C}$-terminal cytoplasmic domain is sensitive to a range of modulators (Pedersen, Riisager, de Paoli, Chen, \& Nielsen, 2016), including nucleotides, such as ATP and NAD+, or regulatory proteins such as PKC (Bennetts et al., 2005, 2012; Hsiao et al., 2010; Tseng et al., 2011). The mutation p.Leu861Pro falls within the putative ATP binding site in CBS2, including residues Glu865 and His847 and Leu848 (Bennetts et al., 2012; Tseng et al., 2011).

Among mammalian CLC proteins, the skeletal-muscle chloride channel CIC-1 has the longest C-terminal peptide after the second CBS domain (Park \& MacKinnon, 2018). Our results show that the C-peptide is important for $\mathrm{CIC}-1$ function and expression. Mutation of Pro883 to Thr induced a dramatic reduction of chloride current amplitude due to a positive shift of voltage dependence of common gating. Interestingly, a similar effect has been described for the nearby p.Ala885Pro mutation identified in the myotonic goat, which generated chloride currents with reduced macroscopic amplitude and altered gating (Beck et al., 1996; Macias et al., 2007). Through functional, biochemical, and NMR spectroscopy studies, these authors demonstrated that the $\mathrm{C}$-terminal region encompasses a conserved segment that folds with a secondary structure containing a short type II poly-proline helix. The flexibility of the last part of the conserved segment appeared critical for channel function. The myotonia-causing mutation p.Ala885Pro extended the poly-proline helix, likely causing a reduction of flexibility of this region. We can thus hypothesize that Pro to Thr mutation in 883 position may also disturb C-terminal flexibility, thereby altering voltage dependence.

Besides, C-peptide also plays an important role in CIC-1 trafficking to the sarcolemma. Previous studies suggested that mutations identified in the most distal part of this region (e.g., p.Arg894stop and p.Pro932Leu) reduced plasma membrane expression (Macias et al., 2007). The results obtained with p.Val947Glu fully agreed with this hypothesis, since this mutation reduced chloride current amplitude without altering the voltage dependence of open probability.

Altogether, the functional characterization of $M C$ mutations allowed us to define the specific role of CBS2 subdomains: mutations between 829 and 835 residues may induce an alteration of voltage dependence, mutations between 851 and 859 residues may produce nonfunctional channels, whereas mutations on 861 residue display no apparent change in $\mathrm{CIC}-1$ (Figure 6). The functional analysis of a higher number of mutations is warranted to confirm this structure-function correlation.

In conclusion, the present study expands the spectrum of CLCN1 mutations responsible for $\mathrm{MC}$ and clarifies the role of the $\mathrm{C}$-terminal region in $\mathrm{ClC}-1$ expression and gating. The comprehension of the molecular mechanisms underlying $\mathrm{MC}$ together with improved understanding of the structure-function relationship of CIC-1 protein could help the discovery of new drugs targeting specific mutant channels' defects, allowing the development of a personalized treatment for $\mathrm{MC}$ patients (Imbrici et al., 2016a).

\section{ACKNOWLEDGMENTS}

We thank the families for participating to this study. We also thank the Naples Human Mutation Gene Biobank (NHMGB), member of the Telethon Network of Genetic Biobanks and of Eurobiobank, for providing us with specimens.

\section{DISCLOSURE STATEMENT}

The authors declare no conflict of interest.

\section{REFERENCES}

Beck, C. L., Fahlke, C., \& George, A. L. Jr. (1996). Molecular basis for decreased muscle chloride conductance in the myotonic goat. Proceedings of the National Academy of Sciences of the USA, 93(20), 11248-11252.

Bennetts, B., Rychkov, G. Y., Ng, H. L., Morton, C. J., Stapleton, D., Parker, M. W., \& Cromer, B. A. (2005). Cytoplasmic ATP-sensing domains regulate gating of skeletal muscle $\mathrm{ClC}-1$ chloride channels. Journal of Biological Chemistry, 280(37), 32452-32458.

Bennetts, B., \& Parker, M. W. (2013). Molecular determinants of common gating of a ClC chloride channel. Nature Communications, 4, 2507.

Bennetts, B., Yu, Y., Chen, T. Y., \& Parker, M. W. (2012). Intracellular $\beta$-nicotinamide adenine dinucleotide inhibits the skeletal muscle ClC-1 chloride channel. Journal of Biological Chemistry, 287, 25808-25820.

Brugnoni, R., Kapetis, D., Imbrici, P., Pessia, M., Canioni, E., Colleoni, L., ... Mantegazza, R. (2013). A large cohort of myotonia congenita probands: novel mutations and a high-frequency mutation region in exons 4 and 5 of the CLCN1 gene. Journal of Human Genetics, 58, 581-587.

Desaphy, J.-F., Gramegna, G., Altamura, C., Dinardo, M. M., Imbrici, P., George, A. L. Jr., ... Conte Camerino, D. (2013). Functional characterization of $\mathrm{ClC}-1$ mutations from patients affected by recessive myotonia congenita presenting with different clinical phenotypes. Experimental Neurology, 248, 530-540. 
Estévez, R., Pusch, M., Ferrer-Costa, C., Orozzo, M., \& Jentsch, T. J. (2004). Functional and structural conservation of CBS domains from CLC chloride channels. Journal of Physiology, 557(Pt 2), 363-378.

Fahlke, C., Beck, C. L., \& George, A. L. Jr. (1997). A mutation in autosomal dominant myotonia congenita affects pore properties of the muscle chloride channel. Proceedings of the National Academy of Sciences of the USA, 94(6), 2729-2734.

Feng, L., Campbell, E. B., Hsiung, Y., \& MacKinnon, R. (2010). Structure of a eukaryotic CLC transporter defines an intermediate state in the transport cycle. Science, 330, 635-641.

Fialho, D., Schorge, S., Pucovska, U., Davies, N. P., Labrum, R., Haworth, A., \& Hanna, M. G. (2007). Chloride channel myotonia: Exon 8 hot-spot for dominant-negative interactions. Brain, 130, 3265-3274.

Ginanneschi, F., Mignarri, A., Lucchiari, S., Ulzi, G., Comi, G. P., Rossi, A., \& Dotti, M. T. (2017). Neuromuscular excitability changes produced by sustained voluntary contraction and response to mexiletine in myotonia congenita. Neurophysiologie Clinique/Clinical Neurophysiology, 47(3), 247-252.

Hebeisen, S., Biela, A., Giese, B., Muller-Newen, G., Hidalgo, P., \& Fahlke, C. (2004). The role of the carboxyl terminus in CIC chloride channel function. Journal of Biological Chemistry, 279(13), 13140-13147.

Hebeisen, S., \& Fahlke, C. (2005). Carboxy-terminal truncations modify the outer pore vestibule of muscle chloride channels. Biophysical Journal, 89(3), 1710-1720.

Hsiao, K.-M., Huang, R.-Y., Tang, P.-H., \& Lin, M.-J. (2010). Functional study of CLC-1 mutants expressed in Xenopus ocytes reveals that a C-terminal region Thr891-Ser892-Thr893 is responsible for the effects of protein kinase $C$ activator. Cellular Physiology and Biochemistry, 25, 687-694.

Imbrici, P., Altamura, C., Camerino, G. M., Mangiatordi, G. F., Conte, E., Maggi, L., ... Camerino, D. C. (2016b). Multidisciplinary study of a new $\mathrm{ClC}-1$ mutation causing myotonia congenita: $\mathrm{A}$ paradigm to understand and treat ion channelopathies. The FASEB Journal, 30(10), 32853295.

Imbrici, P., Altamura, C., Pessia, M., Mantegazza, R., Desaphy, J.-F., \& Conte Camerino, D. (2015a). CIC-1 chloride channels: State-of-the-art research and future challenges. Frontiers in Cellular Neuroscience, 9, 156.

Imbrici, P., Liantonio, A., Camerino, G. M., De Bellis, M., Camerino, C., Mele, A., ... Conte, D. (2016a). Therapeutic approaches to genetic ion channelopathies and perspectives in drug discovery. Frontiers in Pharmacology, 7, 121.

Imbrici, P., Maggi, L., Mangiatordi, G. F., Dinardo, M. M., Altamura, C., Brugnoni, R., ... Camerino, D. C. (2015b). ClC-1 mutations in myotonia congenita patients: Insights into molecular gating mechanisms and genotype-phenotype correlation. Journal of Physiology, 593(18), 41814199.

Koch, M. C., Steinmeyer, K., Lorenz, C., Ricker, K., Wolf, F., Otto, M., ... Jentsch, T. J. (1992). The skeletal muscle chloride channel in dominant and recessive human myotonia. Science, 257(5071), 797-800.

Kornak, U., Kasper, D., Bösl, M. R., Kaiser, E., Schweizer, M., Schulz, A., ... Jentsch, T. J. (2001). Loss of the ClC-7 chloride channel leads to osteopetrosis in mice and man. Cell, 104(2), 205-215.

Lee, T. T., Zhang, X. D., Chuang, C. C., Chen, J. J., Chen, Y. A., Chen, S. C., ... Tang, C. Y. (2013). Myotonia congenita mutation enhances the degradation of human CLC-1 chloride channels. Plos One, 8(2), e55930.

Lehmann-Horn, F., JurKat-Rott, K., \& Rüdel, R. (2008). Diagnostics and therapy of muscle channelopathies - Guidelines of the UIm Muscle Centre. Acta Myologica, 27, 98-113.

Liu, X. L., Huang, X. J., Shen, J. Y., Zhou, H. Y., Luan, X. H., Wang, T., ... Cao, L. (2015). Myotonia congenita: Novel mutations in CLCN1 gene. Channels (Austin), 9(5), 292-298.
Lo Monaco, M., D'Amico, A., Luigetti, M., Desaphy, J.-F., \& Modoni, A. (2015). Effect of mexiletine on transitory depression of compound motor action potential in recessive myotonia congenita. Clinical Neurophysiology, 126(2), 399-403.

Ma, L., Rychkov, G. Y., Hughes, B. P., \& Bretag, A. H. (2009). Analysis of carboxyl tail function in the skeletal muscle $\mathrm{Cl}$ - channel hClC-1. International Journal of Biochemical and Cellular Biology, 41(6), 1402-1409.

Macias, M. J., Teijido, O., Zifarelli, G., Martin, P., Ramirez-Espain, X., Zorzano, A., ... Estevez, R. (2007). Myotonia-related mutations in the distal C-terminus of $\mathrm{ClC}-1$ and $\mathrm{ClC}-0$ chloride channels affect the structure of a poly-proline helix. Biochemical Journal, 403, 79-87.

Markovic, S., \& Dutzler, R. (2007). The structure of the cytoplasmic domain of the chloride channel $\mathrm{CIC}$-Ka reveals a conserved interaction interface. Structure, 15(6), 715-725.

Meyer, S., \& Dutzler, R. (2006). Crystal structure of the cytoplasmic domain of the chloride channel CIC-0. Structure, 14(2), 299-307.

Nielsen, O. B., De Paoli, F. V., Riisager, A., \& Pedersen, T. H. (2017). Chloride channels take center stage in acute regulation of excitability in skeletal muscle: Implications for fatigue. Physiology (Bethesda), 32(6), 425-434.

Park, E., \& MacKinnon, R. (2018). Structure of the CLC-1 chloride channel from Homo sapiens. Elife, 7, e36629. https://doi.org/ 10.7554/eLife.36629

Pedersen, T. H., Riisager, A., de Paoli, F. V., Chen, T. Y., \& Nielsen, O. B. (2016). Role of physiological $\mathrm{ClC}-1 \mathrm{Cl}$ - ion channel regulation for the excitability and function of working skeletal muscle. Journal of General Physiology, 147(4), 291-308.

Portaro, S., Altamura, C., Licata, N., Camerino, G. M., Imbrici, P., Musemuci, O., ... Desaphy, J.-F. (2015). Clinical, molecular, and functional characterization of CLCN1 mutations in three families with recessive Myotonia Congenita. Neuromolecular Medicine, 17, 285-296.

Pusch, M., Steinmeyer, K., Koch, M. C., \& Jentsch, T. J. (1995). Mutations in dominant human myotonia congenita drastically alter the voltage dependence of the CIC-1 chloride channel. Neuron, 15(6), 1455-1463.

Ronstedt, K., Sternberg, D., Detro-Dassen, S., Gramkow, T., Begemann, B., Becher, T., ... Fahlke, C. (2015). Impaired surface membrane insertion of homo- and heterodimeric human muscle chloride channels carrying amino-terminal myotonia-causing mutations. Scientific Report, 5, 15382.

Shalata, A., Furman, H., Adir, V., Adir, N., Hujeirat, Y., Shalev, S. A., \& Borochowitz, Z. U. (2010). Myotonia congenita in a large consanguineous Arab family: Insight into the clinical spectrum of carriers and double heterozygotes of a novel mutation in the chloride channel CLCN1 gene. Muscle Nerve, 41(4), 464-469.

Simon, D. B., Bindra, R. S., Mansfield, T. A., Nelson-Williams, C., Mendonca, E., Stone, R., ... Lifton, R. P. (1997). Mutations in the chloride channel gene, CLCNKB, cause Bartter's syndrome type III. Nature Genetics, 17(2), 171-178.

Skálová, D., Zídková, J., Voháňka, S., Mazanec, R., Musova, Z., Vondracek, P., ... Fajkusova, L. (2013). CLCN1 mutations in Czech patients with myotonia congenita, in silico analysis of novel and known mutations in the human dimeric skeletal muscle chloride channel. Plos One, 8(12), e82549.

Statland, J. M., Bundy, B. N., Wang, Y., Rayan, D. R., Trivedi, J. R., Sansone, V. A., ... Hanna, M. G. (2012). Mexiletine for symptoms and signs of myotonia in nondystrophic myotonia: A randomized controlled trial. Journal of the American Medical Association, 308(13), 1357-1365.

Suetterlin, K. J., Bugiardini, E., Kaski, J. P., Morrow, J. M., Matthews, E., Hanna, M. G., \& Fialho, D. (2015). Long-term safety and efficacy of mexiletine for patients with skeletal muscle channelopathies. JAMA Neurology. 72(12), 1531-1533. 
Tang, C. Y., \& Chen, T. Y. (2011). Physiology and pathophysiology of CLC1: Mechanisms of a chloride channel disease, myotonia. Journal of Biomedicine and Biotechnology, 685328.

Tricarico, D., Mele, A., Calzolaro, S., Cannone, G., Camerino, G. M., Dinardo, M. M., ... Conte Camerino, D. (2013). Emerging role of calcium-activated potassium channel in the regulation of cell viability following potassium ions challenge in HEK293 cells and pharmacological modulation. PLoS One, 8(7), e69551.

Trip, J., Drost, G., Verbove, D.J, van der Kool, A. J., Kuks, J. B., Notermans, N. C., ... Ginjaar, I. B. (2008). In tandem analysis of CLCN1 and SCN4A greatly enhances mutation detection in families with non-dystrophic myotonia. European Journal of Human Genetics, 16, 921 929.

Tseng, P. Y., Yu, W. P., Liu, H. Y., Zhang, X. D., Zou, X., \& Chen, T. Y. (2011). Binding of ATP to the CBS domains in the C-terminal region of CLC-1. Journal of General Physiology, 137, 357-368.

Ulzi, G., Lecchi, M., Sansone, V., Redaelli, E., Corti, E., Saccomanno, D., ... Lucchiari, S. (2012). Myotonia congenita: Novel mutations in CLCN1 gene and functional characterizations in Italian patients. Journal of Neurological Science, 318(1-2), 65-71.

Vindas-Smith, R., Fiore, M., Vásquez, M., Cuenca, P., Del Valle, G., Lagostena, L., ... Morales, F. (2016). Identification and functional characterization of CLCN1 mutations found in nondystrophic myotonia patients. Human Mutation, 37(1), 74-83.
Warnstedt, M., Sun, C., Poser, B., Escriva, M. J., Tranebjaerg, L., Torbergsen, T., ... Fahlke, C. (2002). The myotonia congenita mutation A331T confers a novel hyperpolarization-activated gate to the muscle chloride channel CIC-1. Journal of Neuroscience, 22(17), 7462-7470.

Weinberger, S., Wojciechowski, D., Sternberg, D., Lehmann-Horn, F., JurkatRott, K., Becher, T., ... Fischer, M. (2012). Disease-causing mutations $\mathrm{C} 277 \mathrm{R}$ and $\mathrm{C} 277 \mathrm{Y}$ modify gating of human $\mathrm{ClC}-1$ chloride channels in myotonia congenita. Journal of Physiology, 590(15), 3449-3464.

Wu, F. F., Ryan, A., Devaney, J., Warnstedt, M., Korade-Mirnics, Z., Poser, B., ... Fahlke, C. (2002). Novel CLCN1 mutations with unique clinical and electrophysiological consequences. Brain, 125(Pt 11), 2392-2407. 